\title{
Distrofia miotónica de Steinert
}

\author{
Alfredo Rosado Bartoloméa,b y Lucía Sierra Santos ${ }^{a, c}$.
}

\begin{abstract}
a Grupo de Trabajo SEMFYC Genética Clínica y Enfermedades Raras.

${ }^{\mathrm{b}}$ Médico de Familia. Centro de Salud Mar Báltico. Dirección Asistencial Este. Servicio Madrileño de Salud. Madrid (España).

c Médico de Familia. Centro de Salud Manzanares El Real. Dirección Asistencial Norte. Servicio Madrileño de Salud. Madrid (España).
\end{abstract}

Correspondencia: Alfredo Rosado Bartolomé. Correo electrónico: alfredorosado76@gmail.com.

Recibido el 24 de septiembre de 2014.

Aceptado para su publicación el 8 de octubre de 2014

\section{RESUMEN}

La complejidad y variabilidad de las manifestaciones de la distrofia miotónica tipo 1 o enfermedad de Steinert plantean dificultades para el seguimiento clínico, descrito como fragmentario, inadecuado o incluso deficiente. Las características más discapacitantes son con frecuencia manifestaciones como la somnolencia diurna excesiva, el cansancio, las digestivas y del comportamiento cogntivo, pero sin embargo son los problemas cardíacos, respiratorios o de la deglución los que reducen la esperanza de vida.

Palabras clave: Distrofia Miotónica. Enfermedades Raras. Enfermedades Neuromusculares.

\section{ABSTRACT}

\section{Steinert myotonic dystrophy}

The complexity and variability of the manifestations of myotonic dystrophy tipe 1, or Steinert disease, pose difficulties for clinical follow-up. This is described as fragmentary, inadequate or even poor. The most disabling features are often manifestations such as excessive daytime sleepiness, fatigue, and digestive and cognitive behavior disorders. But yet it is heart, breathing or swallowing problems that reduce life expectancy.

Key words: Myotonic Dystrophy. Rare Diseases. Neuromuscular Diseases.

\section{INTRODUCCIÓN}

La distrofia miotónica tipo 1 o enfermedad de Steinert (CIE-9: 359.2; CIE-10: G71-1; OMIM 160900) es la distrofia muscular más frecuente en adultos. Las estimaciones de su prevalencia oscilan entre aproximadamente 1:100.000 en algunas áreas de Japón hasta aproximadamente 1:10.000 en Islandia, con una prevalencia en Europa de 3-15 por $100.000^{1}$. Se trata de una alteración autosómica dominante, con expresividad clínica muy variable y penetrancia incompleta, debida a una expansión de la repetición inestable del trinucleótido citosina-timina-guanina [CTG]n, localizada en la región 3' no codificante del cromosoma 19q13.3. Aunque existen casos esporádicos, presenta en ocasiones fenómeno de anticipación, por el que las sucesivas generaciones suelen estar más afectadas que el primer caso familiar y su enfermedad debuta más precozmente.

La distrofia miotónica de Steinert es una enfermedad progresiva y pleótropa que puede afectar a varios sistemas como el muscular, respiratorio, cardíaco, endocrino, ocular y sistema nervioso central. Sus manifestaciones características comprenden una pérdida progresiva de fuerza muscular más distal que proximal, ptosis palpebral, debilidad de los músculos faciales, de la mandíbula y parte anterior del cuello, miotonía, somnolencia diurna, cansancio y cataratas (tabla 1). Hasta la fecha, no existe un tratamiento curativo para esta patología. Habitualmente, los síntomas se hacen más evidentes en el período medio de la vida, pero los signos pueden ser detectables en la primera o segunda década. Se han identificado diferentes fenotipos clínicos según la edad de inicio: congénito (presente al nacer), infantil (inicio antes 


\section{Muscular}

- Atrofia y debilidad muscular en cara y párpados, mandíbula, cuello, antebrazos y manos, parte inferior de las piernas y pies con miotonía (dificultad para la relajación muscular).

- Disartria.
- Dificultad para caminar, pies caídos (equinismo)

- Traspiés y caídas frecuentes.

- Falta de expresión facial.

- Lenguaje mal articulado, voz débil, boca entreabierta.

\section{Sistema nervioso central}

- Cansancio permanente.

- Somnolencia diurna excesiva.

- Falta de iniciativa y apatía.

- Depresión.

- Deterioro cognitivo.

\section{Sistema visual}

- Cataratas precoces (<40 años).

- Ptosis palpebral.

- Visión borrosa o disminución de la visión.

- Los párpados caídos pueden ser un problema para leer o mirar la televisión.

\section{Digestivo}

- Disfagia.

- Gastroparesia.

- Alternancia de diarrea y estreñimiento.

- Abdominalgia frecuente.

- Incontinencia fecal.

- Malnutrición.
- Cambios en la personalidad con falta de motivación, inercia, testarudez y pensamiento repetitivo, lo que puede a su vez causar problemas de relación con la familia, amigos y en la escuela o el trabajo.

\section{Cardiocirculatorio}

- Trastornos del ritmo y la conducción:

- Fibrilación o flúter auricular.

- Bloqueo AV de segundo o tercer grado.

- Ritmo de la unión.

- Intervalo PR > 200 ms.

- Duración QRS > 105 ms.

- Bloqueo de rama derecha con hemibloqueo, especialmente si existe bloqueo AV asociado.

- Bradicardia sinusal sintomática.
- Dificultades para masticar (atrofia de maseteros y temporales) y deglutir, atragantamientos frecuentes (riesgo de aspiración broncopulmonar):

- Ensuciar la ropa interior, especialmente en situaciones de estrés o excitación.

- Síncopes, palpitaciones, taquicardia y, en ocasiones, falta absoluta de sintomatología.

- Hipotensión.

\section{Respiratorio}

- $\quad$ Alteraciones respiratorias relacionadas con el sueño (SAHS).

- Debilidad de musculatura respiratoria.
- Trastornos del sueño, ronquidos, dificultad para despertar, cefalea matutina y somnolencia diurna.

- Dificultad para toser o tos débil.

- Infecciones respiratorias de repetición, inclusive neumonías. Grave riesgo anestésico de broncoaspiración.

Tabla 1. Principales signos y síntomas de la distrofia miotónica tipo 1 o enfermedad de Steinert. Nota: Es muy improbable que un mismo paciente desarrolle todos estos problemas simultáneamente. La relación de sistemas afectados no es exhaustiva. Elaboración propia. AV: Auriculoventricular. SAHS: Síndrome de apnea-hipopnea del sueño. 
de los 10 años de edad), adulto precoz (inicio entre los 11 y 20 años de edad), adulto (inicio de 21 a 40 años de edad) y tardío o parcial (inicio después de los 40 años de edad). Muchos de los rasgos clínicos, metabólicos y endocrinos del fenotipo de la distrofia miotónica tipo 1 afectan habitualmente al anciano normal, pero aparecen a una edad más precoz en los pacientes con enfermedad de Steinert. Entre los signos de envejecimiento acelerado están la relación positiva entre las concentraciones de folitropina (FSH) y lutropina (LH) y el número de repeticiones CTG, las alteraciones de la conducción cardíaca, la resistencia a la insulina e intolerancia a la glucosa, la dislipemia, el bajo nivel de deshidroepiandrosterona (DHEAS), la disfunción eréctil y la calvicie en el hombre, así como la aparición precoz de cataratas entre otras manifestaciones ${ }^{1}$.

\section{CASO CLÍNICO}

Presentamos el caso de un varón de 36 años de edad en 2014 sin antecedentes personales y familiares de interés, que refiere pérdida de fuerza en ambas manos documentada desde los 33 años de edad con antecedentes de fractura traumática de cúbito y radio, reducida mediante cirugía con sedación. Para determinar el origen de la pérdida de fuerza en las manos, el médico de familia solicita una interconsulta a Neurología para excluir una neuropatía compresiva, concretamente un síndrome del túnel del carpo. El neurólogo de zona realiza un estudio electromiográfico (EMG) en mayo de 2011 sin encontrar signos de neuropatía de los nervios mediano derecho y cubital bilateral e identifica una CPK de $281 \mathrm{mU} / \mathrm{ml}$ (normal: 38-174). En julio de 2012, debido al empeoramiento de la debilidad en las manos, y a instancias nuevamente del médico de familia, el mismo neurólogo de zona repite la EMG identificando esta vez un patrón miopático con descargas miotónicas en el extensor común de los dedos, compatible con distrofia miotónica, por lo que el paciente es derivado a neurología del hospital de referencia para confirmación diagnóstica.

En febrero de 2013 se realiza biopsia del deltoides para excluir miopatía, sin obtener un diagnóstico definitivo por muestra insuficiente para el diagnóstico anatomopatológico, con analítica en la que sólo destacan CPK de $343 \mathrm{mU} / \mathrm{ml}$ e $\mathrm{lgG}$ de $506 \mathrm{mg} / \mathrm{dl}$ (normal: 700-1.600). En abril de 2013 se efectúa un estudio de genética molecular, identificándose una expansión del extremo 3'UTR del gen DMPK con 120 repeticiones que confirma el diagnóstico de distrofia miotónica tipo 1 o enfermedad de Steinert.
El paciente es derivado a Cardiología, donde en junio de 2013 se realizó ecocardiografía transtorácica $(E T T)$, en la que se identifica una hipertrofia ventricular izquierda (HVI) ligera y una fracción de eyección del ventrículo izquierdo (FEVI) de $50-55 \%$, a la vez que se practica una resonancia magnética (RM) con contraste, la cual pone de manifiesto una probable miocardiopatía incipiente con un ventrículo izquierdo dilatado y una función sistólica biventricular en el límite bajo para su intervalo de edad, recomendándose control evolutivo en función del contexto clínico. En julio de 2013 se comprueba la existencia en el electrocardiograma (ECG) convencional de bloqueo aurículo-ventricular (BAV) de primer grado con PR 270 ms, QRS 130 ms y alteraciones de la repolarización compatibles con HVI. En agosto de 2013 el Holter ECG constata la existencia de BAV de primer grado con PR máximo de 360 ms, frecuencia cardiaca máxima de 124 latidos por minuto (lpm), mínima nocturna de 33 Ipm con extrasistolia ventricular y supraventricular de baja densidad, sin pausas patológicas. Se recomienda seguimiento en consultas de Cardiología en seis meses.

En el seguimiento neurológico de septiembre de 2013 se identifica una ptosis palpebral que no cubre el borde pupilar, una debilidad bilateral del orbicular y del elevador de los párpados y una disartria leve con un balance motor que muestra debilidad distal $4 / 5$ en miembros inferiores y fenómeno miotónico en manos. En este momento se proporciona asesoramiento genético por Neurología, se facilita información escrita sobre la enfermedad, se sugiere excluir otros casos en la familia y se recomienda estudio prenatal en caso de que el paciente desee tener descendencia. Se objetiva también una pérdida de fuerza muscular proximal y distal tales que, junto con la miotonía, impiden al paciente realizar movimientos finos o rápidos con las manos o cargar pesos, por lo que el médico de familia inicia los trámites correspondientes de forma que en febrero de 2014 se reconoce al paciente una incapacidad permanente total.

En la valoración oftalmológica realizada en enero de 2014 se identifican cataratas incipientes bilaterales. En marzo de 2014 se repiten ETT y Holter ECG y se comprueba una fracción de eyección en el límite inferior de la normalidad y BAV de primer grado con PR máximo de $360 \mathrm{~ms}$. Se indica nueva revisión en seis meses para valorar la implantación de un marcapasos permanente en función del resultado del estudio electrofisiológico.

En septiembre de 2014, en un centro hospitalario 
diferente al de referencia, el paciente se somete a un estudio electrofisiológico (EEF) para valoración de la conducción aurículo-ventricular, así como de inducción de arritmias ventriculares, identificándose un trastorno de conducción nodal y His-Purkinje severos, con disfunción sinusal, ausencia de inducibilidad de arritmias ventriculares sostenidas y función ventricular izquierda en limites bajos de la normalidad. Una semana después, se le implanta marcapasos DDDR, bicameral en la aurícula derecha y el ventrículo derecho, y se le suministra un dispositivo cardioemisor para seguimiento domiciliario.

\section{DISCUSIÓN}

Dificultad y lentitud para alcanzar un diagnóstico definitivo son características habituales de las enfermedades raras. Para ilustrar ambas se enumeran cronológicamente las diferentes posibilidades etiológicas exploradas, así como las pruebas complementarias realizadas, independientemente de su utilidad diagnóstica final. También se ha querido mostrar de qué modo un motivo de consulta de origen aparentemente musculoesquelético conduce al diagnóstico de una enfermedad sistémica y, por último, a la identificación de una cardiopatía grave. El fenómeno miotónico en las manos como motivo de consulta inicial y la edad de diagnóstico de nuestro paciente coinciden con lo descrito en la bibliografía. La edad promedio de inicio de la clínica de la distrofia miotónica de Steinert es de 26,1 \pm 13,2 años. El síntoma de comienzo más frecuente es el agarrotamiento de las manos $(38,3 \%)$ y el dolor se describe como manifestación inicial en el $3 \%$ de los casos. El período de tiempo medio necesario para alcanzar el diagnóstico correcto es de $7,3 \pm 8,2$ años $^{2}$.

Es preciso destacar que la insistencia del médico de familia en busca del origen de la sintomatología fue decisiva, pese a los resultados negativos de la primera EMG, al solicitar una nueva valoración neurológica en la cual se pudo identificar con un elevado nivel de certidumbre la verdadera etiología, lo que a su vez posibilitó que el neurólogo se centrase en la confirmación diagnóstica mediante pruebas complementarias específicas dirigidas.

El carácter complejo y variable de las manifestaciones de la distrofia miotónica tipo 1 dificulta extraordinariamente su seguimiento, que ha sido descrito como fragmentario, inadecuado o incluso deficiente para muchos pacientes. Numerosos factores contribuyen a esta situación: las manifestaciones multisistémicas de la enfermedad, que dan como resultado diferentes discapacidades; el bajo nivel educativo y escasez de ingresos de los pacientes, así como su pobre red de apoyo social; el grado variable de conocimiento de la enfermedad de Steinert entre los médicos; la carencia de una pauta consensuada para la asistencia de los pacientes con esta patología y las diferencias en el acceso y tipo de recursos sanitarios disponibles localmente. Estos factores subrayan la necesidad de un planteamiento terapéutico integral en la asistencia de los pacientes con enfermedad de Steinert basado en la vigilancia de las complicaciones tratables. Es difícil establecer unos criterios clínicos sólidos. En una revisión sistemática de la bibliografía publicada entre 1980 y 2005 sobre el seguimiento clínico de esta enfermedad se comprobó que la mayoría de los artículos publicados sobre prevalencia, métodos de evaluación y procedimientos de intervención si sitúan en la categoría de niveles de evidencia no analíticos o de opinión de expertos ${ }^{3}$.

La inexistencia de procedimientos terapéuticos que modifiquen la evolución de la enfermedad ha dado lugar tradicionalmente a que los pacientes no sean adecuadamente tratados o no reciban seguimiento alguno ${ }^{4}$. Con frecuencia los enfermos, e incluso los propios clínicos, tienen la percepción errónea de que, una vez confirmado el diagnóstico, no es posible hacer nada para mejorar la evolución de la enfermedad. Sin embargo, las evidencias en contra de esta inhibición terapéutica son cada vez más numerosas ${ }^{5}$. Además, se han demostrado unas tasas de prevalencia superiores al doble cuando se aplican técnicas de diagnóstico molecular en comparación con el diagnóstico basado exclusivamente en criterios clínicos ${ }^{6,7}$. Por lo tanto, la utilidad del estudio genético molecular no se limita a la confirmación diagnóstica, sino que puede permitir la identificación, tras el caso índice, de enfermos asintomáticos o paucisintomáticos no exentos de patología, tal como sucede en el caso que se presenta.

Actualmente existe un criterio unánime sobre la necesidad de una vigilancia estricta de los trastornos cardíacos del ritmo y la conducción que cursan de forma silente, de las posibles complicaciones respiratorias graves de procesos infecciosos banales y de los riesgos anestésicos ${ }^{8-10}$. La Scottish Muscle Network ofrece en acceso libre una Guía para Profesionales para el seguimiento de la distrofia miotónica tipo 1 en el adulto elaborada específicamente para médicos de Atención Primaria: http://www. smn.scot.nhs.uk/documents/DM1\%20PROFESSIONALS\%20LEAFLETUpdated\%20June10.pdf. 


\section{BIBLIOGRAFÍA}

1. Turner C, Hilton-Jones D. The myotonic dystrophies: diagnosis and management. J Neurol Neurosurg Psychiatry. 2010; 81 (4): 358-67.

2. Hilbert JE, Ashizawa T, Day JW, Luebbe EA, Martens WB, McDermott MP et al. Diagnostic odyssey of patients with myotonic dystrophy. J Neurol. 2013; 260 (10): 2497-504.

3. Gagnon C, Chouinard MC, Laberge L, Veillette S, Bégin P, Breton $\mathrm{R}$ et al. Health supervision and anticipatory guidance in adult myotonic dystrophy type 1 . Neuromuscul Disord. 2010; 20 (12): 847-51.

4. Hilton-Jones D. Myotonic dystrophy. Forgotten aspects of an often neglected condition. Curr Opin Neurol. 1997; 10 (5): 399-401.

5. Johnson NE, Heatwole CR. Myotonic dystrophy: from bench to bedside. Semin Neurol. 2012; 32 (3): 246-54.

6. Kamsteeg EJ, Kress W, Catalli C, Hertz JM, WitschBaumgartner M, Buckley MF et al. Best practice guidelines and recommendations on the molecular diagnosis of myotonic dystrophy types 1 and 2. Eur J Hum Genet. 2012; 20 (12): 1203-8.

7. Siciliano G, Manca M, Gennarelli M, Angelini C, Rocchi A, ludice $A$ et al. Epidemiology of myotonic dystrophy in Italy: re-appraisal after genetic diagnosis. Clin Genet. 2001; 59 (5): 344-9.

8. de Die-Smulders CE, Höweler CJ, Thijs C, Mirandolle JF, Anten B, Smeets $\mathrm{HJ}$ et al. Age and causes of death in adult-onset myotonic dystrophy. Brain. 1998; 121 (Pt 8): 1557-63.

9. Wahbi K, Meune C, Porcher R, Bécane HM, Lazarus A, Laforêt $P$ et al. Electrophysiological study with prophylactic pacing and survival in adults with myotonic dystrophy and conduction system disease. JAMA. 2012; 307 (12): 1292301.

10. Bennum M, Goldstein B, Finkelstein Y, Jedeikin R. Continuous propofol anaesthesia for patients with myotonic dystrophy. Br J Anaesth. 2000; 85 (3): 407-9. 